\title{
Dynamic Channel Flow Control of Networks-on-Chip Systems for High Buffer Efficiency
}

\author{
Sung-Tze Wu, Chih-Hao Chao, I-Chyn Wey, and An-Yeu (Andy) Wu \\ Graduate Institute of Electronics Engineering and Department of Electrical Engineering \\ National Taiwan University \\ Taipei, Taiwan, 10617, R.O.C. \\ Contact information: cason@access.ee.ntu.edu.tw;
}

\begin{abstract}
System-on-Chip (SoC) designs become more complex nowadays. The communication between each processing element often suffers challenges due to the wiring problem. Networks-on-Chip (NoC) provides a practical solution to solve the problem. The major components in NoC are routers, which are dominated by the buffer size. Previous mechanisms need large buffer size to achieve high performance. In this paper, a dynamic channel flow control mechanism is proposed to realize the channel resource sharing globally, which can increase the throughput and the channel utilization rate. An $8 \times 8$ mesh on-chip network is implemented on a cycle accurate simulator. By the experimental result, the proposed mechanism can reduce the buffer size by $30 \%$ as compared with virtual channel flow control at the same throughput. Moreover, the throughput can be improved by $20 \%$ as compared with wormhole flow control.
\end{abstract}

Index Terms - Flow control, Networks-on-chip.

\section{INTRODUCTION}

System-on-Chip (SoC) designs provide a possible and economical method to integrate complex systems on a single chip with the advanced VLSI technology. However, the exponential growth in speed and integration levels of intellectual properties (IPs) has increased the interconnection complexity, which dominates the design and its performance $[14,16]$. The major problems caused by the interconnection wires are the wiring complexity, the global synchronization difficulty, and limitation of transmission bandwidth $[14,16]$. To deal with the on chip interconnection problems, a new methodology, Networks-on-Chip (NoC), has been developed for the next-generation SoC paradigm $[2,3,8,12]$. NoC acts as a high-performance, chip-level communication infrastructure with high regularity and modularity [2, 3]. The wire complexity in a point-to-point interconnection can be reduced by this new interconnection infrastructure. It also

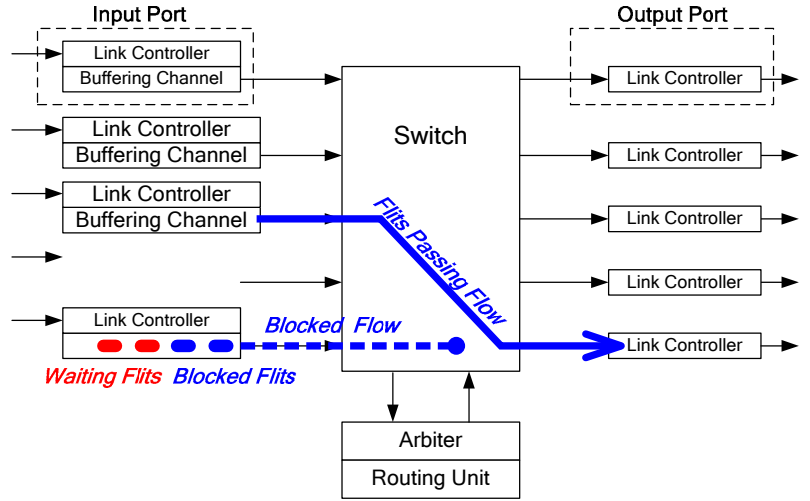

Fig. 1: A packet suffers the HoL problem in a conventional wormhole router.

solves the problem of scalability of the SoC bus $[2,3,16]$.

The NoC architecture is constructed by routers. Moreover, the flow control mechanism between routers dominates the communication of the whole network [11]. A good flow control mechanism shall utilize the limited bandwidth of links to satisfy the diverse applications and guarantee quality-of-service (QoS) with minimal buffering overhead [1]. Store-and-forward flow control [5] (SAF) is the simplest method to establish data transmissions by storing a complete packet in a large buffer. To reduce the buffer size, however, wormhole flow control [13] slices the packet into flits. Nevertheless, wormhole flow control suffers the head-of-line (HoL) blocking problem. Virtual channel flow control was proposed to solve the HoL blocking problem [4]. This paper proposes a dynamic channel flow control to further improve the channel flexibility by sharing the channel resource globally. In the mechanism, dynamic channels serve as a highly flexible channel. Dynamic channels can be allocated by any input port of the router to substitute the unavailable input virtual channel. In this way, dynamic channel flow control can increase the channel flexibility and enhance the buffer utilization. Simulation results show that the proposed dynamic channel flow control not only increases the throughput of the whole NOC by $20 \%$ as compared with 


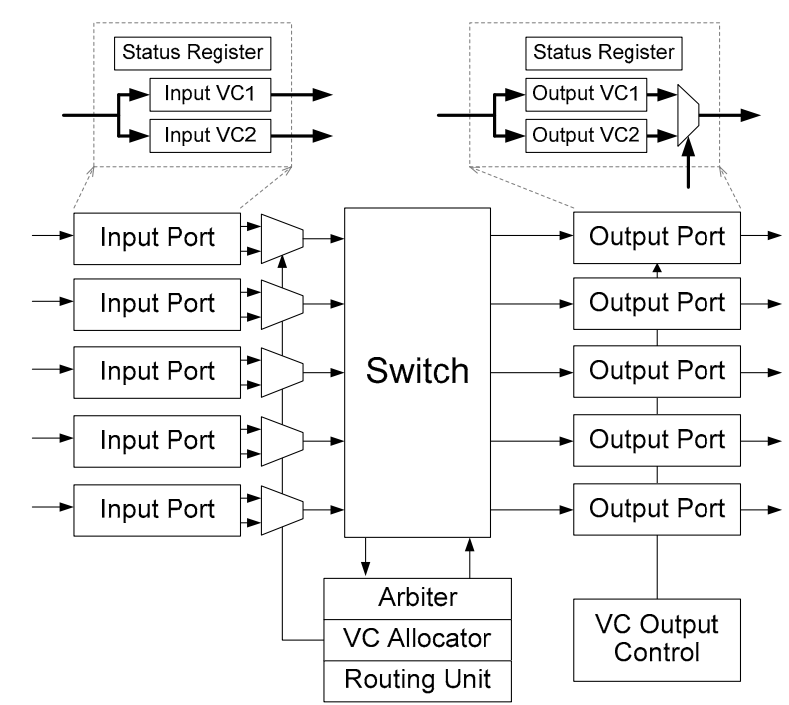

Fig. 2: The functional block diagram of a virtual channel router

wormhole flow control, but also improves the buffer utilization by $19.6 \%$ as compared with virtual channel flow control.

Section II reviews the previous flow control mechanisms. Section III introduces dynamic channel flow control mechanism and its architecture. Section IV shows our experimental results with a cycle-accurate simulation model. Finally, we conclude our work in section V.

\section{RELATED WORKS}

Wormhole flow control [13] was first introduced to accomplish a fast single-chip implementation of the router and reduced the buffer requirement by splitting the packet into flits. The channel assignment is allocated in the packet-level and the packet transmits in the flit-level. A head flit acts as a packet to allocate the physical channel in advance. Body flits pass directly through the channel in the flit-level. The channel is free to use until the tail flit crosses. For this reason, a fixed amount of the storages also result in lower latency and greater throughput because some flits of a packet are accepted before an entire packet buffer is available. However, the HoL blocking problem frequently occurs inside the wormhole router [5]. The problem makes the flits to span multiple routers and leads to heavy traffic contention. Fig. 1 shows the HoL problem in the wormhole router. The blue thick dot line represents packets which are blocking in a channel. These flits of the packet are blocked due to the channel is used by the packet. Thus, the new coming flits, the red thick dot line, cannot deliver to other output channel through this channel.

To conquer the HoL problem, there are several works applying virtual channel flow control in the router design for NoC [10]. Virtual channel flow control [4] has been proposed to solve the problem and to increase the channel utilization and the throughput. In these works, virtual channels are dedicated to the input ports. Fig. 2 shows the block diagram of a virtual channel router. Each port is associated to several virtual channels. An output channel of a packet is determined by the virtual channel allocator with the head flit. When a virtual channel is blocked, other flits can still traverse the physical channel through the other virtual channels. In the study of flit-reservation flow control [11], the author separates control flits and data flits. The routing operations can be performed before arriving of data flits. Control flits traverse the network before the data flits to reserve buffers of the channel. Data flits are forwarded or buffered through the reserving channels. This provides an efficient use of the buffers and bandwidth to reduce the latency.

\section{Proposed Dynamic ChanNEl Flow CONTROL MECHANISM}

In traditional flow control, although a virtual channel is free to use, the other blocked input port cannot use the channel. Second, some virtual channels in a router are not connected to any router in a mesh topology; these channels are underutilized. This work introduces resource sharing methodology by extracting virtual channels from the port-level to the router-level. Dynamic channel flow control is proposed to improve the channel flexibility. The channel utilization rate and the throughput of a router can be enhanced greatly by sharing few dynamic channels. Therefore, the dynamic channels are put to serve flexible channels for heavy loading of the traffic. Fig. 3 shows a generic router to suffer the HoL blocking problem. A packet comes to the input port but channels are not available to use. The packet can not advance due to the buffer full in a virtual channel router. In the proposed dynamic channel flow control, channels in a router are made more flexible compared to the channel in an input port. The packet can be dynamically redirected to these dynamic channels to prevent HoL blocking problem. Hence, the channel utilization rate can be increased by sharing channels for a router.

\subsection{Hardware Architecture}

Fig. 4 shows the functional block diagram of proposed dynamic channel flow control. The proposed flow control relaxes virtual channels from each input port to share with all of the input ports. However, each input port in a dynamic channel router can still have dedicated channels for the average bandwidth requirement. A pseudo port, called dynamic port, collects the extra sharable channels, called dynamic channels, for the router. These channels unlike virtual channels serve for a dedicated input port. When the dedicated channel in an input port is busy for serving the packet, a dynamic channel is going to act the channel for that port. These dynamic channels serve as the virtual channel for an unavailable input channel. 


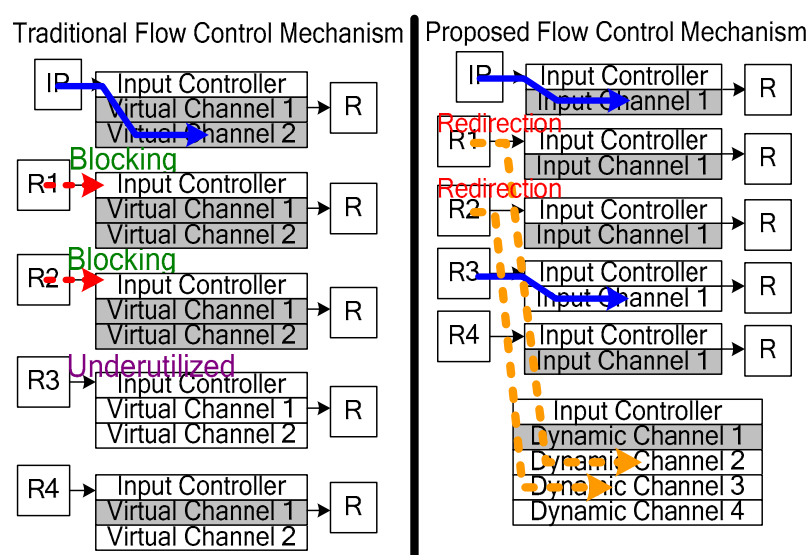

Fig. 3: An extra channel solving the blocking problem increases the throughput.

The replacement control unit performs the channel replacement is based on our channel replacement policy. The channel replacement is determined by the information stored in the control register and the status register. Control registers and status registers hold for the each dynamic channel status to help with the decision of the packet advancement. The control registers save the input port number where the packet comes, and the status registers store the channel status and the allocation information in regard to the output channel.

Each dynamic channel needs to be numbered a unique channel ID to identify the channel number for channel allocation. The packet of dynamic channel flow control is attached a channel ID field into the head flit for allocating the dynamic channel or the dedicated channel. Each of the input port can have the same number of dedicated channels to satisfy the average bandwidth requirement of the network traffic load. The channel ID of each dedicated channel can be independently numbered and the maximum channel ID of each input port would be the same. The channel ID of dynamic channels should number over the number of the dedicated channel.

The proposed dynamic channel flow control router is implemented in $\mathrm{C}++$ model and runs on our simulator for NoC. The detailed router architecture is shown in Fig. 6.

\subsection{Operations}

\subsubsection{Input Operation}

Dynamic channel flow control implements to support input ports from four directions. The local port is not supported in the current sharing mechanism. This can drastically decrease the traffic load of input ports by dispatching some of packets to dynamic channels. Fig. 5 shows the router operations of the proposed dynamic channel flow control mechanism. As the input part shown, the send router decides to use a dedicated channel or a dynamic channel for the packet advancement. When the dedicated channel of the input port is allocating to another

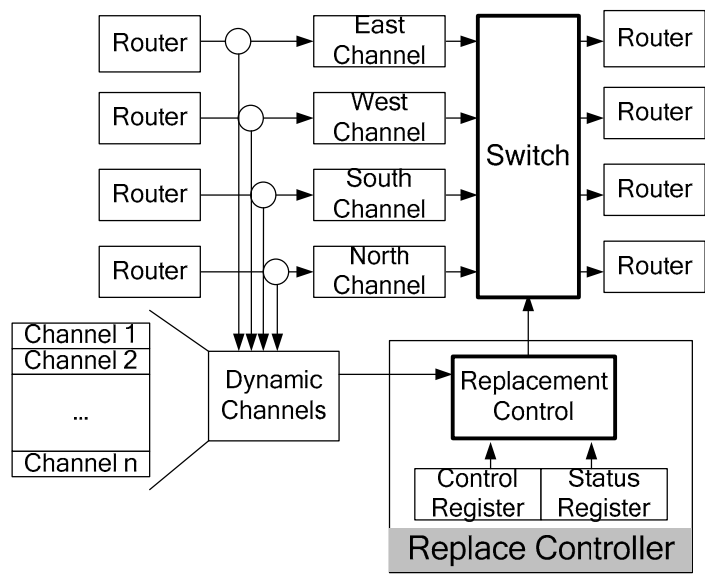

Fig. 4: The functional block diagram of the proposed dynamic channel flow control

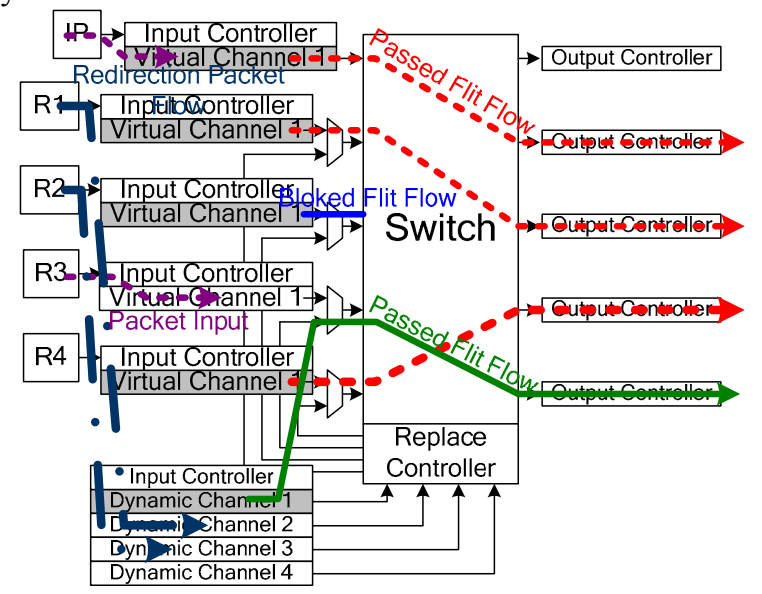

Fig. 5: The operations of a router implementing the proposed dynamic channel flow control.

packet to use, the send node can direct the packet to a dynamic. The channel allocation mechanism is used by the proposed mechanism to process the injected packets at input ports of the switch.

\subsubsection{Output Operation}

However, when a dynamic channel is occupied by a packet, the dynamic channel needs to replace a channel of the input port to advance the packet to next hop. The dynamic channel needs to compete with the generic input port the access right to the output channel when the dynamic channel holds a packet. The replacement of a dynamic channel is competed with the original channel. That can have two situations as following:

The first case is to grab the unused to substitute. In this case, the output channel can directly authorize the dynamic channel to access until the whole packet transmission is done. In fact, a router at the corner of the topology can always get a free channel to replace, for example mesh topology. Consequently, the channel utilization rate can increase by replacing these channels. Second one is to compete the use right. The input port needs to use the output channel to deliver its packet. In this 


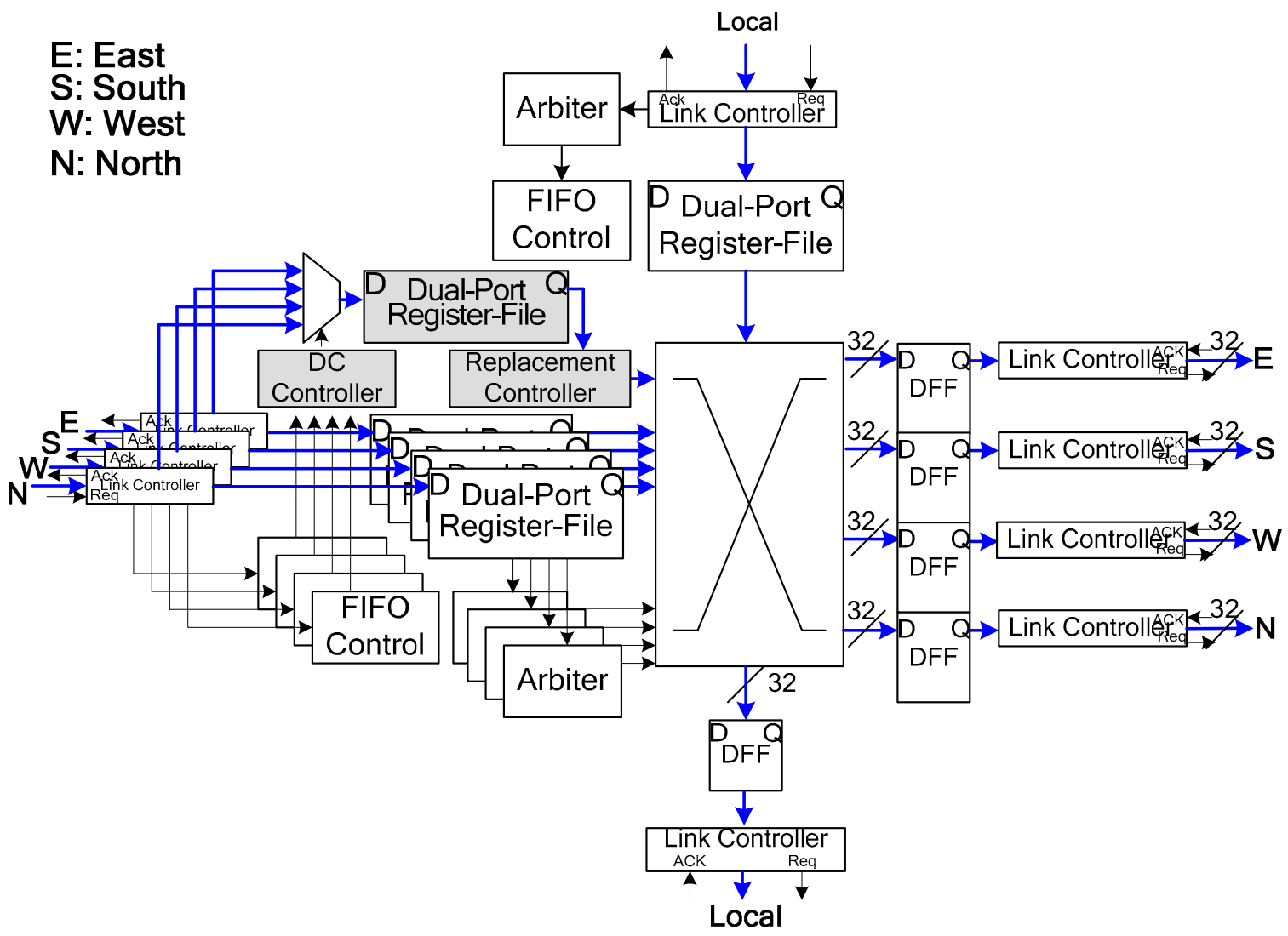

Fig. 6: The detailed router blocks of the proposed dynamic channel flow control.

case, the output channel should be scheduled for both the dynamic channel and the dedicated channel in the input port with a scheduling algorithm. In our implementation, the age control mechanism is used for the priority decision. The older packet can have the highest priority to use the channel to deliver flits. Any arbitration algorithm, however, can be used to schedule the channel including the round-robin arbitration, the random arbitration, or the priority arbitration [13].

\section{EXPERIMENTAL RESULTS}

A cycle-accurate simulator is implemented to measure the performance of on-chip network. The simulator models a 2-dimension $8 \times 8$ mesh as the network topology. For comparison, the conventional wormhole flow control and the virtual channel flow control are also implemented in the simulator. Each node injects 1000 packets to the network in the warm-up phase. The network goes into the stable state, each node generates 10000 packets and injects to the network for the data analysis. We analyse the metrics of each flow control mechanisms such as latency, throughput, etc.. The simulator adopts $\mathrm{XY}$ routing algorithm [5] and deadline algorithm [5] for channel selection to implement the router. Also, an iSLIP algorithm [15] is referred to implement our scheduling algorithm. Our router model assumes that it takes one clock cycle for a packet to cross a link and takes another clock cycle to model the internal delay [13]. Each router in the network has five input and five output ports to interconnect other routers as the 2D mesh topology. The router has a set of 4 flits in a buffer per channel. Setups with different number of channels are simulated to reveal the performance.

Fig. 7 shows the average latency of wormhole flow control and dynamic channel flow control as a function of the offered traffic. Wormhole flow control saturates at $40 \%$ capacity in the network. Our proposed dynamic channel flow control extends the throughput to $50 \%$. Thus, dynamic channel flow control improves wormhole flow control $10 \%$ with a dynamic channel to be added. the number of dynamic channels is increased to compare the effect of channel number, With two dynamic channels, the throughput raises to $55 \%$. Adding one dynamic channel can further improve one dynamic channel by $5 \%$ and improves wormhole by $15 \%$. Finally, we place 4 dynamic channels into the router and the throughput is enhanced around $65 \%$. Thus, comparing results with different number of channels that each added dynamic channel improves around 5\%.

Fig. 8 shows the comparison on average latency between virtual channel flow control and proposed dynamic channel flow control at different constant traffic injection rates. The proposed flow control with 36 flits per router reduces the average latency of virtual channel flow control 


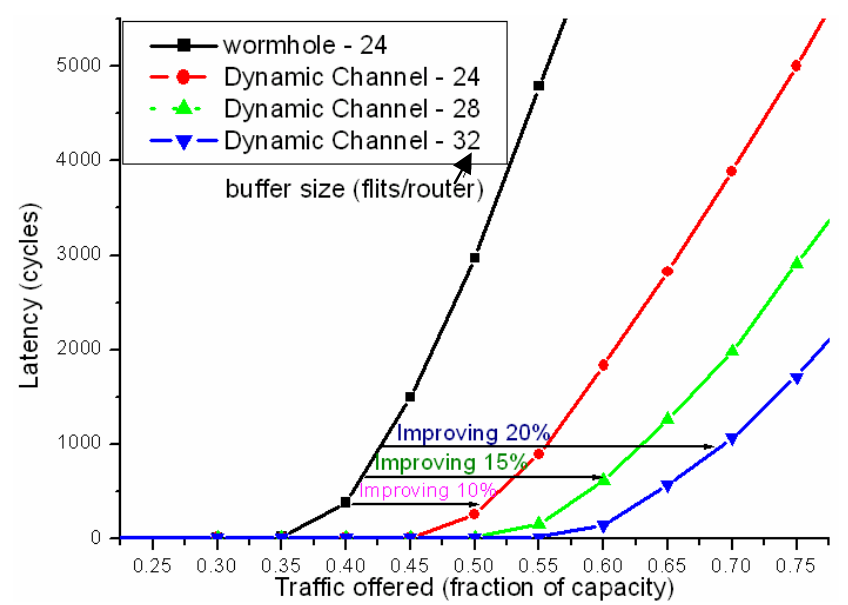

Fig. 7: Average latency comparison of flow control mechanisms in generic wormhole flow control and dynamic channel flow control with different channels on the $8 \times 8$ mesh topology.

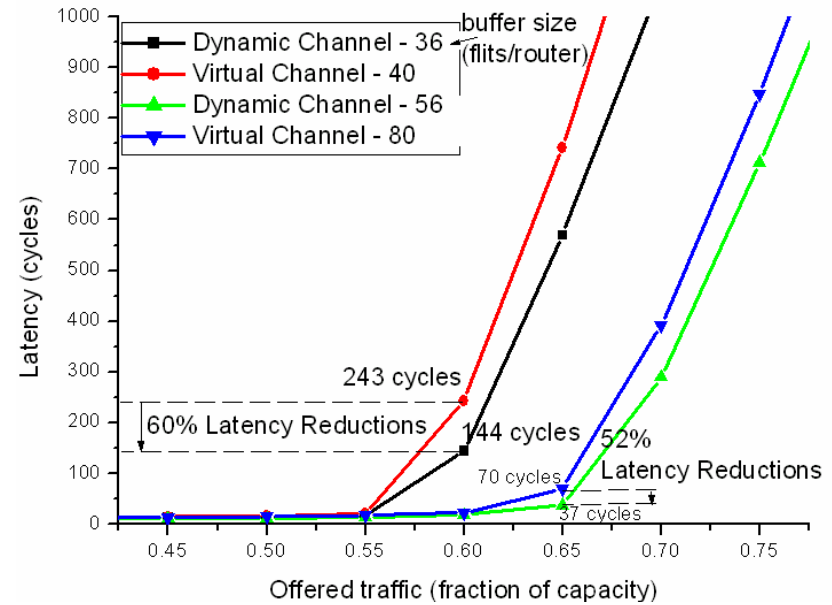

Fig. 8: Average latency comparison of the generic virtual channel flow control and the proposed dynamic channel flow control simulated on the 8 x 8 mesh topology.

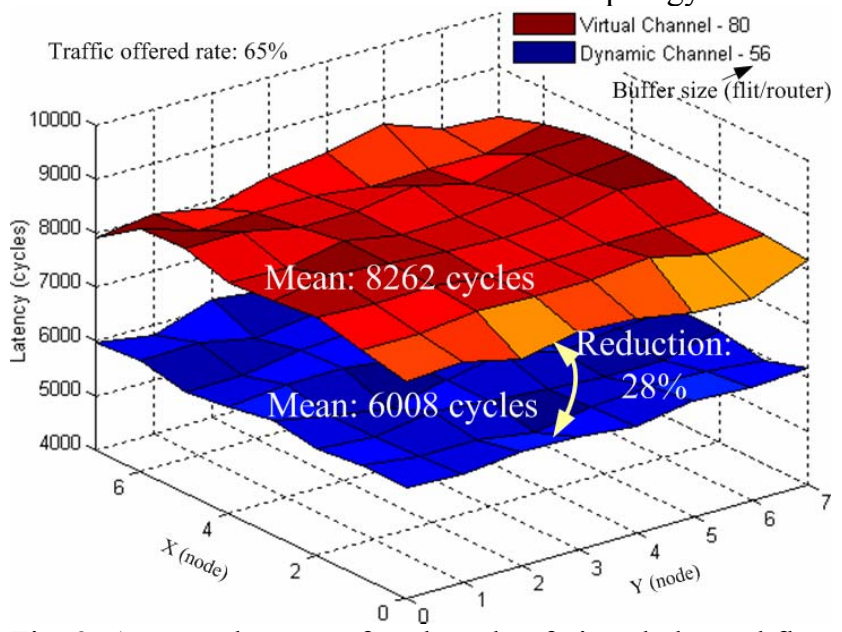

Fig. 9: Average latency of each node of virtual channel flow control and dynamic channel flow control simulated on the $8 \times 8$ mesh topology.

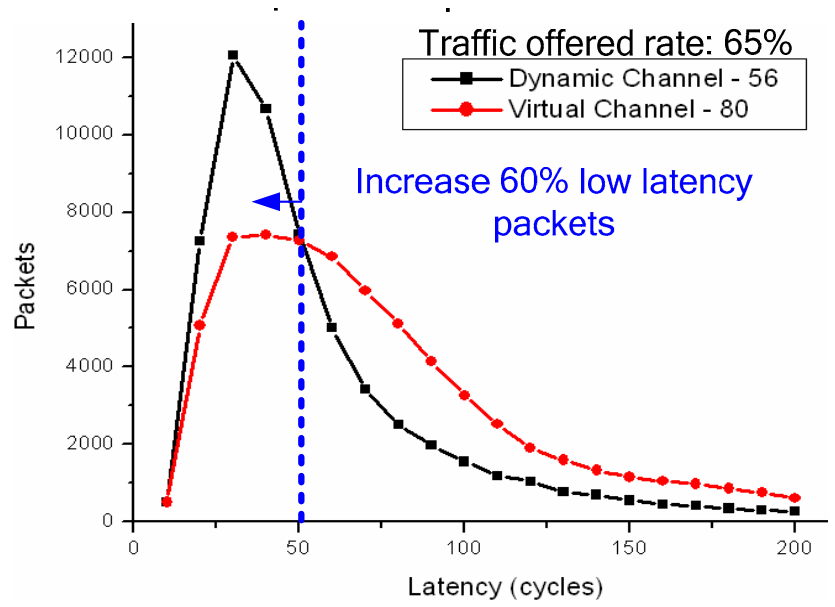

Fig. 10: The latency distribution of packets simulated on the $8 \times 8$ mesh with traffic offered rate $65 \%$.

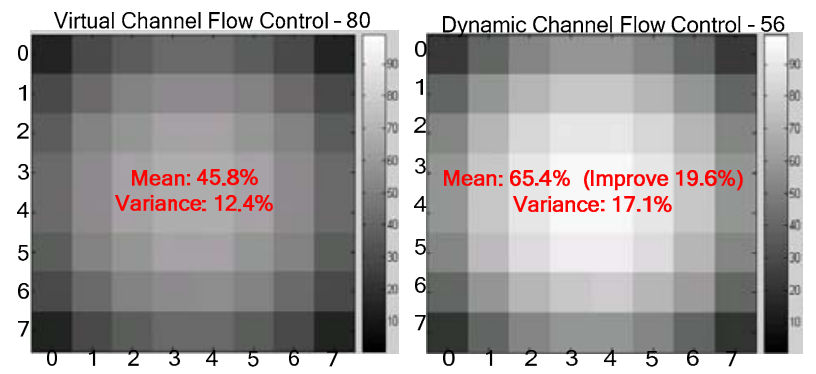

Fig. 11: The buffer utilization for the different flow control mechanisms simulated at traffic offered rate $65 \%$.

with 40 flits per router by $60 \%$ at $60 \%$ traffic capacity. The proposed dynamic channel flow control has high efficiency of the channel buffer and low the transmission latency in comparison with the virtual channel flow control at the same buffer size and channel numbers. The proposed dynamic channel flow control reduces the buffer requirement by $11 \%$ under the same throughput comparing with the virtual channel flow control in Fig. 8. Moreover, the Fig. 8 also shows the result that adds the number of the dedicated channel for an input port into the dynamic channel to run the simulation. Our dynamic channel flow control can have more flexible ability to increase the channel utilization rate, and the router can get a better performance improving. The dynamic channel flow control with 56 flits per router reduces the buffer requirement by $30 \%$, and the average latency can further decrease by $52 \%$ by increasing the channel utilization rate comparing virtual channel flow control with 80 flits per router.

Fig. 9 shows the average latency of each node on the network simulated on the traffic offered rate at $65 \%$. As the result shows the proposed dynamic channel flow control sums to the average mean value with 6008 cycles and virtual channel flow control has the average mean value with 8262 cycles. Our proposed dynamic channel flow control can reduce virtual channel flow control by $28 \%$. 
Fig. 10 shows the latency distribution simulated at the traffic injection rate $65 \%$. Our proposed dynamic channel flow control can largely decrease the transmission time. As the result shows in Fig. 10, packets which the transmission time is less than 50 cycles can be increased by $60 \%$ as compared with virtual channel flow control. Our proposed dynamic channel flow control further reduces the packet counts that have large transmission time. Thus, the packets occupy a channel with the shorter time.

Fig. 11 shows the buffer utilizations of different flow control mechanisms for each node at the traffic offered rate $65 \%$. The virtual channel flow control associates more virtual channels with the physical channel to enhance the channel utilization. However, virtual channel flow control also induces most packets to be trapped in HoL blocking when packets are injecting quickly to the buffer. Hence, The proposed dynamic channel flow control use the flexible channel dispensed mechanism to provide the service for the packet and gets good buffer utilizations. The buffer utilization of dynamic channel flow control is better than virtual channel flow control with $19.6 \%$. The proposed dynamic channel flow control reduces the buffer requirement and uses smaller buffer size to achieve the same throughput and lower transmission latency.

\section{CONCLUSiOnS}

In this paper, a flow control mechanism is proposed for future NoC-based SoC design. The dynamic channel flow control adopts an efficient and flexible channel sharing technique. Compared with existing flow control mechanisms, the proposed mechanism uses dynamic channels to improve the channel flexibility. The proposed dynamic channel flow control can decrease the buffer requirements. The experimental result shows that throughput is improved by $20 \%$ compared with the wormhole flow control. At the same traffic capacity, the proposed dynamic channel flow control can reduce the latency by $60 \%$ compared with the virtual channel flow control with the same buffer size.

\section{REFERENCES}

[1] E. Bolotin, I. Cidon, R. Ginosar, and A. Kolodny, "QNoC: QoS architecture and design process for Network on Chip," Journal of Systems Architecture, Dec. 2003.

[2] L. Benini, and G. D. Micheli, "Networks on Chips: A New SoC Paradigm," Journal of IEEE Computer, vol. 35, pp. 70-78, Jan. 2002.

[3] W. J. Dally, and B. Towles, "Route Packets, Not Wires: On-Chip Interconnection Networks," In Proceedings of the 38th Design Automation Conference, pp. 684-689, Jun. 2001.
[4] W. J. Dally, "Virtual-channel flow control," IEEE Transactions on Parallel and Distributed Systems, vol. 3, pp. 194-205, Mar. 1992.

[5] J. Duato, S. Yalamanchili, and L. Ni, Interconnection Networks, Morgan Kaufmann, 2002.

[6] P. Guerrier, and A. Greiner, "A generic architecture for on-chip packet-switched interconnections," In Proceedings of Design, Automation and Test in Europe Conference and Exhibition 2000, pp. 250-256, Mar. 2000.

[7] A. Jantsch, and H. Tenhunen, Networks on Chip, Kluwer, 2003.

[8] S. Kumar, et al, "A Network on Chip Architecture and Design Methodology," IEEE Computer Society Annual Symposium on VLSI, pp. 105-112, Apr. 2002.

[9] G. Moore, "No Exponential Is Forever: But Forever Can Be Delayed," In Proceedings of 2003 IEEE International Solid-State Circuits Conference, vol. 1, pp. 20-23, 2003.

[10] N. Kavaldjiev, G. J. M. Smit, and P. G. Jansen, "A virtual channel router for on-chip networks," In Proceedings of the IEEE International SOC Conference, Sept. 2004.

[11] Li-Shiuan Peh, and W. J. Dally, "Flit-reservation flow control," Sixth International Symposium on High-Performance Computer Architecture, pp. 73-84, Jan. 2000.

[12] M. Sgroi, et al, "Addressing the System-on-a-Chip Interconnect Woes Through Communication-Based Design," In Proceedings of the 38th Design Automation Conference, pp. 667-672, Jun. 2001.

[13] B. Towles, and W. J. Dally, Principles and Practices of Interconnection Networks, Morgan Kaufmann, 2004.

[14] Ron Ho, et al, "The Future of Wires," Proceedings of the IEEE, vol. 89, pp. 490-504, 2001.

[15] N. McKeown, "The iSLIP scheduling algorithm for input-queued switches" IEEE/ACM Transactions on Networking, vol. 7, pp. 188-201, 1999.

[16] K. Lahiri, et al, "Design of High-Performance System-on-Chips using Communication Architecture Tuners," IEEE Transaction on CAD/ICAS, vol. 23, pp. 620-636, 2004. 